\title{
Physics analysis of the ITER ECW system for an optimized performance
}

\author{
G Ramponi', D Farina' ${ }^{1}$, M A Henderson ${ }^{2}$, E Poli ${ }^{3}$, O Sauter ${ }^{2}$, G Saibene ${ }^{4}$, H \\ Zohm $^{3}$ and C Zucca ${ }^{2}$ \\ ${ }^{1}$ Istituto di Fisica del Plasma, CNR, EURATOM-ENEA-CNR Ass., Milano, Italy \\ ${ }^{2}$ CRPP, EURATOM-Confédération Suisse, EPFL, CH-1015, Lausanne, Switzerland \\ ${ }^{3}$ IPP-Garching, Max-Planck-Institut für Plasmaphysik, D-85748 Garching, Germany \\ ${ }^{4}$ EFDA Close Support Unit, Boltzmannstrasse 2, D-85748 Garching, Germany
}

\begin{abstract}
A predictive analysis of the capabilities of the ITER Electron Cyclotron Wave system is presented in this work. Modifications of both the Upper Launcher and the Equatorial Launcher aimed at increasing the potentialities of the system are identified and discussed. A modification of the Upper Launcher has been already incorporated in the updated front steering design called Extended Physics Launcher. By adopting different deposition ranges for the upper and lower steering mirrors, this design offers the flexibility to drive current from the radial region required for the stabilization of neoclassical tearing modes up to that required for sawtooth control, allowing a synergy with the Equatorial Launcher. Here a comparison of the performances of the new design of the Upper Launcher with those of a Dropped Upper Launcher is performed, showing that better performances for both NTM stabilization and sawtooth control may be obtained by lowering the upper port location. An analysis of the EC current driven by the Equatorial Launcher is also presented, showing that adding to the present design the possibility to drive counter-current in addition to the existing co-current capability would significantly increase the flexibility of the system. The behaviour of all launchers at reduced magnetic fields is also discussed.
\end{abstract}

PACS numbers: $52.35 . \mathrm{Hr}, 52.50 . \mathrm{Sw}$, 52.55.Fa

\section{Introduction}

The ITER Electron Cyclotron Wave (ECW) system consists of up to 24, $170 \mathrm{GHz}$ gyrotrons, 1 to 2 MW each, connected to one launcher situated in one equatorial port (EL) and four launchers situated in the upper ports (UL), for a nominal injected power of $20 \mathrm{MW}$. Key objectives of the whole system are central heating, q profile control and stabilization of magnetohydrodynamic (MHD) instabilities, mainly Neoclassical Tearing Modes (NTM) and sawteeth. The two launchers systems have different current drive characteristics: the EL, where the beams are steered in the toroidal direction, gives broad driven current density $\left(j_{\mathrm{CD}}\right)$ profiles and high driven current $\left(\mathrm{I}_{\mathrm{cd}}\right)$, good for central deposition and current profile control, while the UL, where the beams are steered in the poloidal direction, may allow narrow $j_{\mathrm{CD}}$ profiles, good for controlling MHD activity such as NTM and sawteeth. However, to fulfil all the abovementioned goals, both systems should be optimized such as to cover adequately the largest operational domain possible.

We recall that up to the end of 2005 the exclusive goal assigned to the UL (based on three upper ports) was NTM stabilization, while all other functions were ascribed to the EL. Detailed beam tracing calculations have been carried out by means of two beam tracing codes, GRAY [1] and TORBEAM [2], with the goal of evaluating the performance of the UL for NTM stabilization considering a number of different mm-wave designs proposed for the Remote Steering (RS) and the Front Steering (FS) launcher options, as part of EFDA technology tasks [3]. The analysis of all launcher design 
options has been based on three selected H-mode scenarios (scenarios 2, 3 and 5) having considerably different $q, T, n$ and bootstrap current profiles at one time slice (End Of Burn or EOB). To account for the uncertainties in the detailed predictions of the ITER plasma profiles, both the equilibrium database and the analysis have been extended to include variations of the poloidal beta $\beta_{\mathrm{p}}$ and of the internal inductance $l_{i}$ representative of typical variations observed in JET and ASDEX Upgrade H-modes with $q_{95}$ between 3 and 4 . Only the two modes having the most severe effects on plasma confinement, namely $(2,1)$ and $(3,2)$, have been included in the analysis. The radial range of interest, representative of likely locations of the $3 / 2$ and $2 / 1 \mathrm{q}$ surfaces in most NTM-prone ITER plasmas, turned out to be from $\rho_{\text {tor }} \sim 0.52$ to $\rho_{\text {tor }} \sim 0.85$ ( $\rho_{\text {tor }}$ being the square root of the normalized toroidal flux). This set of conditions established the access requirements for the UL, and provided the basis for the definition of the steering range. Considering that the main effect of ECCD in stabilizing the neoclassical tearing modes is to drive a helical current within the island to compensate the missing bootstrap current $j_{b s}$, $\eta_{N T M}=j_{E C C D} / j_{b s}$ has been defined as the NTM stabilization efficiency. Further, we adopted a criterion to evaluate the launcher performance based on a fit to experimental data [4], i.e $\eta_{N T M}$ has to be above 1.2 at $\mathrm{q}=2$ and $\mathrm{q}=3 / 2$ flux surfaces of all the $\mathrm{H}$-mode reference scenarios considered. The analysis showed that, provided an 'optimal' fixed toroidal injection angle and well collimated beams are used, the FS launcher exceeds the requirements by a large margin, bringing the power requirement down to $2 / 3$ of the installed $20 \mathrm{MW}$ [5].

Since the FS design offers appreciable reserve in $\eta_{N T M}$, a new variant of the UL has been designed that, by adopting different deposition ranges for upper and lower mirrors, offers the flexibility to increase the access range beyond that required by the NTM stabilisation, thus allowing a synergy between UL and EL [6]. Besides providing good values of $\eta_{N T M}$, this new variant, called Extended Physics Launcher (EPL), allows to drive well-localized current density up to the $q=1$ location. This makes it attractive for additional applications besides active NTM stabilization, as, for instance, plasma current tailoring, control of a $(4,3)$ mode in order to achieve a "Frequently Interrupted Regime" (FIR), and sawtooth control. In the FIR regime the growth of $(3,2)$ NTM is often interrupted by ergodization due to its nonlinear coupling to an ideal $(4,3)$ mode. On ASDEX Upgrade it has been shown that lowering the magnetic shear with co-ECCD just outside the radius of the $q=4 / 3$ flux surface triggers the transition to this regime [7]. Sawtooth activity is expected to be of primary importance for ITER, where, due to the stabilizing effect of alpha particles, long periods sawteeth are expected to occur [8]. The crashes events terminating the long sawtooth free periods can provide 'seed islands' for the NTMs [9]. Therefore sawteeth destabilization, i.e., shortening the sawtooth period, may prevent destabilization of the NTMs. It has been also shown, both theoretically and experimentally. that localized co-CD just inside (or counter-CD just outside) the $\mathrm{q}=1$ surface may shorten the sawtooth period $[10,11]$. The effectiveness in controlling the sawtooth period depends on the capability of ECCD to modify the evolution of the magnetic shear at the $q=1$ surface, i.e. on the radial derivative of the driven current profile [12].

All this leads to the conclusion that the efficiency for all the objectives of the EPL increases with more localized, higher current density profiles. A larger efficiency means that less power is required to fulfil the objective. Minimizing the EC power is in itself an important issue for ITER since this will limit the deterioration of the fusion gain Q [13]. The optimization of the EC current driven by the EPL has been carried out with respect to the injection angles and the beam parameters, by considering the present upper port location. However, additional optimization of the driven current density profiles may be obtained by lowering the upper port location, due to a greater localization occurring when the EC beam travels tangentially to the flux surface in the neighbourhood of the absorption region $[14,15]$. Here a variant of the EPL is considered. We refer to this variant in terms of Dropped Upper Launcher (DUL), which is similar to the EPL except for the fact that it is lowered by approximately $65 \mathrm{~cm}$ (one blanket shield module).

As far as the Equatorial Launcher is concerned, we point out two possible modifications of the present design. Note that one of the main applications foreseen for the EL is central heating, that might be required, for example, to go above the L-H threshold or to control the density peaking during the early stage of the scenario, as currently done in present experiments [16]. Presently central heating can only be provided with co-current drive (co-CD), that might lead to excessive peaking of the current density profile in the standard scenario 2, to early onset of $q=1$ surface in the hybrid scenario 3 or even to 
prevent the development of steady state scenario 4. Extending the present design so as to provide also counter-CD possibility would allow to compensate the modification of the plasma current profile, as useful when only heating is required. Moreover, this would improve the flexibility of the system for central q control, since three actuators, i.e. central co-ECCD, counter-ECCD and heating would be made available. An additional useful modification is the possibility of launching poloidally tilted beams from the top and low steering mirrors in order to improve their capability of accessing the core plasma region.

The paper is organized as follows. In Section 2 a comparison of the efficiencies for NTM stabilization and sawteeth control of the EPL and the DUL is presented. In Sec. 3 the capabilities of the EL are shown and the potential effects of central co- and counter-ECCD on the q profile of the inductive scenario 2, the hybrid scenario 3, and the reverse shear scenario 4 are discussed. In Sec. 4 an overview of the capabilities of all launchers at reduced magnetic fields is presented. Finally, the last section is devoted to some concluding remarks.

\section{Comparison of the EPL and DUL performances}

The EPL consists of four launchers, each housing eight beams, and two steering mechanisms, positioned at different heights in the present upper port plug. The two steering mirrors are identified as Upper Steering Mirror (USM) and Lower Steering Mirror (LSM). Since each steering mirror carries 4 beams, each set of 4 mirrors controls up to 16 beams, i.e. up to $13.3 \mathrm{MW}$. The lower set is dedicated to cover the outer region of the plasma, i.e., $0.55 \leq \rho_{\text {tor }} \leq 0.85$, and the upper set to access radial range $0.3 \leq \rho_{\text {tor }} \leq 0.8$. In the region of overlap, the injected power may reach $20 \mathrm{MW}$. The main physics objectives of this launcher are NTM stabilization and sawtooth control.

As already mentioned, the optimization study for the EPL has been carried with respect to both the toroidal injection angles, to find the 'optimal' $\mathrm{n}_{/ /}$, and with respect to the beam parameters, in order to find the 'optimal' beam size, since both contribute to optimize the current density profiles. Using well collimated beams with focus inside the plasma and 'optimal' toroidal injection angles, values in excess of the requirements in the figures of merit for both NTM stabilization and sawteeth control have been achieved [17]. Here, considering that additional optimization of the driven current density profiles is obtainable lowering the launching location, namely using the 'Dropped Upper Launcher' (DUL), we shall compare the performances of the two launchers in terms of the figures of merit of for their main physics objectives.

The ECCD calculations by GRAY code have been carried out for both steering mirrors of the EPL and the DUL having the following locations: EPL_LSM: $(\mathrm{R}, \mathrm{Z})=(6.90 \mathrm{~m}, 4.18 \mathrm{~m})$; DUL_LSM= $(\mathrm{R}$, $\mathrm{Z})=(7.70 \mathrm{~m}, 3.55 \mathrm{~m})$; EPL_USM: $(\mathrm{R}, \mathrm{Z})=(6.85 \mathrm{~m}, 4.39 \mathrm{~m})$; DUL_USM= $(\mathrm{R}, \mathrm{Z})=(7.56 \mathrm{~m}, 3.73 \mathrm{~m})$. The same circular, well-collimated beams, proved to be 'optimal' for EPL, have been used to compare the two launchers: the beam launched from the LSM locations has its waist $w_{0}=2.1 \mathrm{~cm}$ at a distance $+1.62 \mathrm{~m}$ from the steering mirror (+ means towards the plasma) while that launched from the USM has its waist $w_{0}=2.9 \mathrm{~cm}$ at a larger distance from the mirror $(+2.13 \mathrm{~m})$. The beams are launched with a toroidal angle $\beta=20^{\circ}$ (except for EPL_USM where $\beta=18^{0}$ ), and are steered varying the poloidal angle $\alpha$. The comparison is performed keeping the range of radial locations that has been chosen for the EPL.

Figures 1 and 2 show the peak values of the current density profiles, their normalized full widths at $1 / \mathrm{e}\left(\Delta \rho_{t o r}\right)$, the driven currents and the poloidal steering ranges for USM and LSM injection into the plasma of scenario 2 (EOB2). The better performance of the DUL, mainly for current driven at innermost surfaces, is evident. This is due both to an increment of the total driven current related to optimal values of $\mathrm{n}_{/ /}$and a better relation between the launcher location and the geometry of flux surfaces providing higher and narrower current density profiles. In the case of USM (see figure 1), an increase of the driven current density by a factor up to 2.6 (at $\rho_{\text {tor }}=0.3$ ) and a substantial reduction of the profile width (up to a factor 0.5) are obtained. We note, however, that a larger steering range is required for the DUL to cover the same radial region as covered by the EPL. For instance, while with the DUL_USM the steering range required to drive current from $\rho_{\text {tor }}=0.8$ to $\rho_{\text {tor }}=0.3$ is $\Delta \alpha=36^{0}$ is, with the EPL_USM $\Delta \alpha=24^{0}$ is sufficient.

In the case of the LSM (figure 2), an exception to the favourable behaviour of the DUL is observed for $\rho_{\text {tor }} \geq 0.8$. This is mainly due to the fact the power absorption in that radial region occurs at larger 
distances from the DUL_LSM with respect to EPL_LSM, so that the 'local' spot size of the beam launched from DUL_LSM is larger than the 'local' spot size of the same beam when launched from EPL_LSM. This may be deduced by looking at beam trajectories by the LSM of both DUL and EPL shown in figure 3. By taking this into account, a different beam, with focus at a larger distance from the LSM, should be used for a better performance of DUL at outermost flux surfaces. We further note that, if the EC power is injected from the DUL_LSM, low $\alpha$, 'quasi horizontal' beam trajectories are needed to get ECCD at the outermost surfaces. These trajectories are affected by high refraction that, in its turn, affects the local beam size making the beam astigmatic. These observations indicate that a specific optimization study would be required for the DUL to assess its performances at outermost surfaces, by taking also into account the possibility of exchanging the roles of the two steering mechanisms.
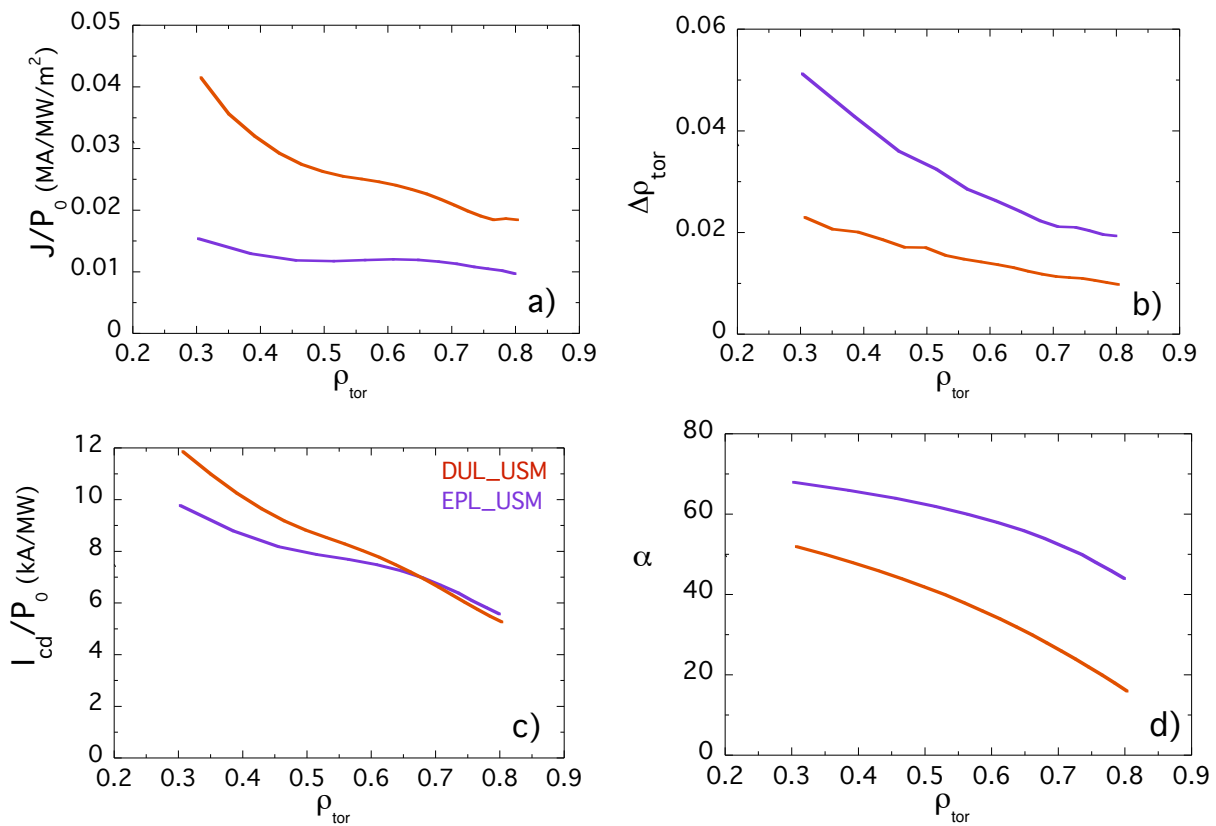

Figure 1. Peak of the driven current density a), normalized width at $1 / \mathrm{e} \Delta \rho_{\text {tor }}$ b), total driven current $\mathrm{c}$ ), and poloidal steering angle d) as a function of the radial location $\rho_{\text {tor }}$ for $1 \mathrm{MW}$ of injected power into the plasma of Scenario 2 (EOB2) from the USM of the DUL (red curve) and of the EPL (blue curve) with toroidal injection angle $\beta=20^{\circ}$. 

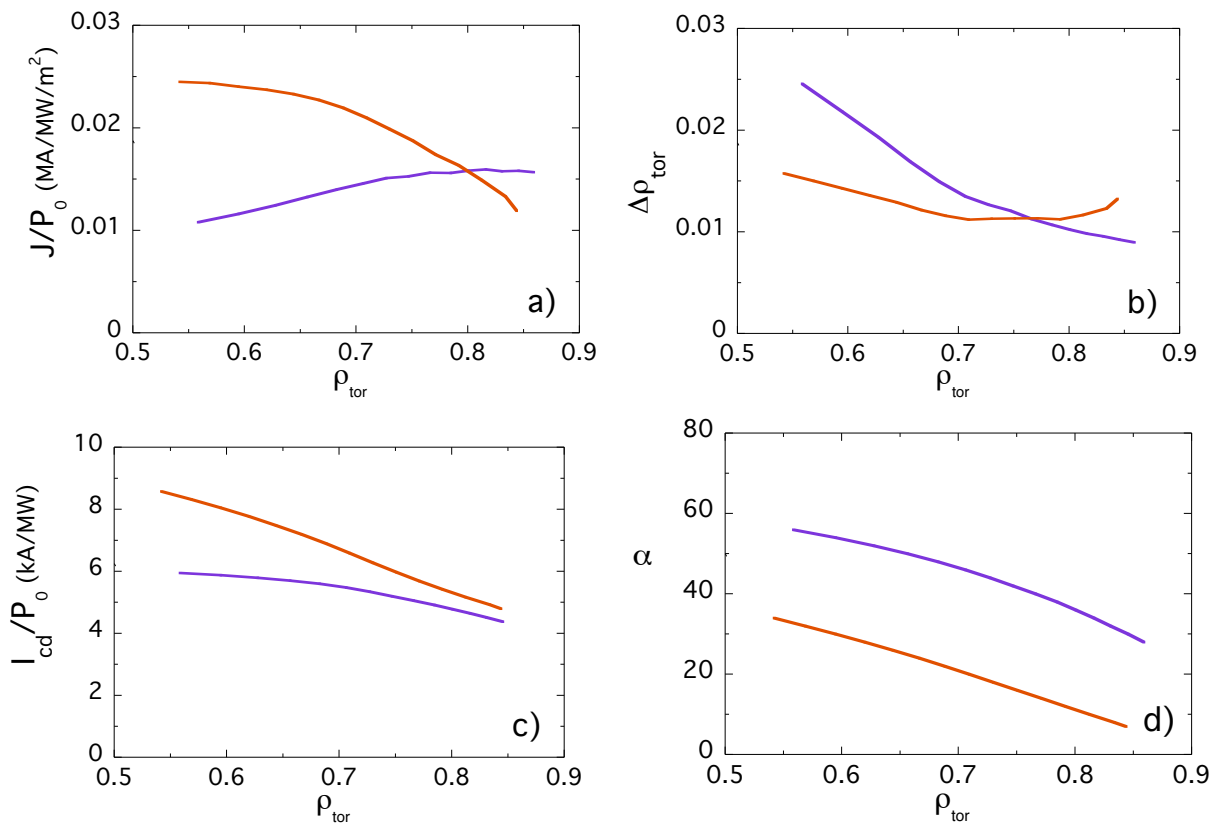

Figure 2. Same as figure 1, for injection from the LSM of the DUL at $\beta=20^{\circ}$ and from the LSM of the EPL at $\beta=18^{0}$.
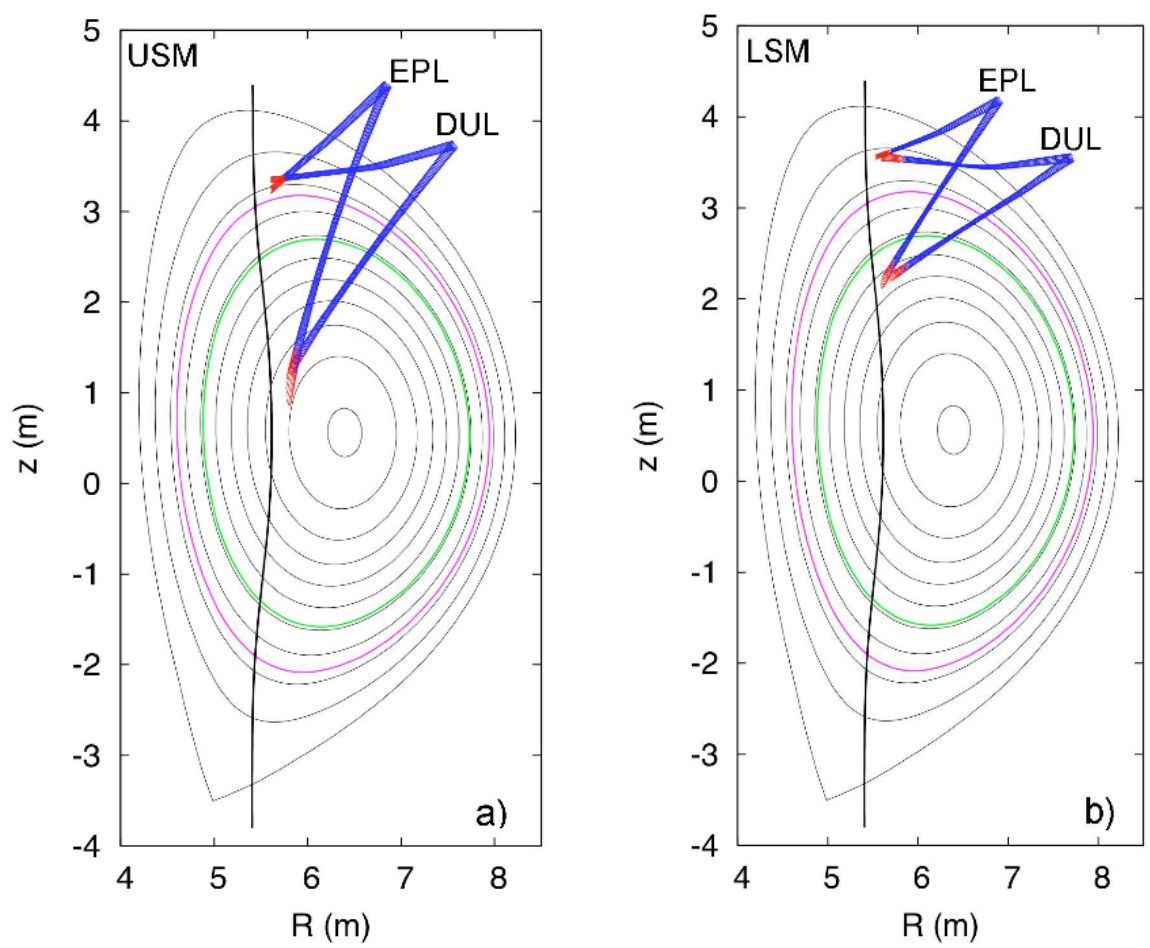

Figure 3. Beam trajectories of the wave beam injected into the plasma of scenario 2 from USM a) and from LSM b), aiming to drive current at the extrema of the corresponding radial range, for both EPL and DUL. The pink and green lines denote the $\mathrm{q}=2$ and $\mathrm{q}=3 / 2$ flux surfaces, respectively.

In order to compare of the efficiencies of the two launchers for NTM stabilization with localized ECCD, the three H-mode reference scenarios, i.e., the inductive scenario 2 (EOB2), the hybrid scenario 3 (EOB3), and the low q scenario 5 (EOB5) have been considered. The main parameters of the scenarios are summarized in Table 1. 
Table 1. Parameters of the ITER H-mode reference scenarios

\begin{tabular}{lllllllll}
\hline Scenario & $\begin{array}{l}\mathrm{I}_{\mathrm{p}} \\
(\mathrm{MA})\end{array}$ & $\beta_{\mathrm{N}}$ & $\begin{array}{l}\mathrm{n}_{\mathrm{e} 0} \\
\left(10^{20} \mathrm{~m}^{-3}\right)\end{array}$ & $\begin{array}{l}\mathrm{T}_{\mathrm{e} 0} \\
(\mathrm{keV})\end{array}$ & $\rho_{\text {tor } 2 / 1}$ & $\begin{array}{l}\mathrm{J}_{\mathrm{bs}}\left(\rho_{2 / 1}\right) \\
\left(\mathrm{MA} / \mathrm{m}^{2}\right)\end{array}$ & $\rho_{\text {tor } 3 / 2}$ & $\begin{array}{l}\mathrm{J}_{\mathrm{bs}}\left(\rho_{3 / 2}\right) \\
\left(\mathrm{MA} / \mathrm{m}^{2}\right)\end{array}$ \\
\hline EOB2 & 15 & 1.8 & 1.02 & 24.8 & 0.75 & 0.073 & 0.62 & 0.094 \\
EOB3 & 12 & 2.2 & 0.86 & 31.8 & 0.67 & 0.120 & 0.56 & 0.147 \\
EOB5 & 17 & 2.1 & 1.24 & 29.5 & 0.78 & 0.096 & 0.67 & 0.111
\end{tabular}

The values of the figure of merit for $(2,1)$ and $(3,2)$ NTM stabilization are shown in Tables 2 and 3 for 13.3 MW of EC power injected respectively from the EPL_LSM and from the DUL_LSM. Besides $\eta_{N T M}$, the full widths, $w_{c d}$, of the EC current profiles (with $w_{c d}=a \Delta \rho_{t o r}$ and $a=200 \mathrm{~cm}$ the plasma minor radius), and the products $\eta_{N T M} w_{c d}$ reflecting the effective total driven current are quoted in the Tables.

Table 2. NTM figures of merit for EPL_LSM

\begin{tabular}{ccccc}
\hline Scenario & $\mathrm{q}$ & $\eta_{\mathrm{NTM}}$ & $\mathrm{w}_{\mathrm{cd}}(\mathrm{cm})$ & $\eta_{\mathrm{NTM}} \mathrm{w}_{\mathrm{cd}}$ \\
\hline EOB2 & 2 & 2.8 & 2.4 & 6.7 \\
& $3 / 2$ & 1.8 & 3.8 & 6.8 \\
EOB3 & 2 & 2.0 & 3.0 & 6.0 \\
& $3 / 2$ & 1.3 & 4.7 & 6.1 \\
EOB5 & 2 & 1.7 & 2.0 & 3.4 \\
& $3 / 2$ & 1.4 & 2.8 & 3.9 \\
\hline
\end{tabular}

Table 3. NTM figures of merit for DUL_LSM

\begin{tabular}{ccccc}
\hline Scenario & $\mathrm{q}$ & $\eta_{\mathrm{NTM}}$ & $\mathrm{w}_{\mathrm{cd}}(\mathrm{cm})$ & $\eta_{\mathrm{NTM}} \mathrm{w}_{\mathrm{cd}}$ \\
\hline EOB2 & 2 & 3.4 & 2.3 & 7.6 \\
& $3 / 2$ & 3.4 & 2.7 & 9.0 \\
EOB3 & 2 & 3.2 & 2.2 & 7.1 \\
& $3 / 2$ & 3.0 & 2.9 & 8.5 \\
EOB5 & 2 & 1.5 & 2.7 & 4.0 \\
& $3 / 2$ & 2.0 & 2.5 & 5.0 \\
\hline
\end{tabular}

Looking at Tables 1 and 2, the following observations can be made: i) all $\eta_{N T M}$ values are above 1.2 for both launchers; ii) in the case of the DUL, larger $\eta_{N T M}$ values (by a factor from 1.2 at $\mathrm{q}=2$ of scenario 2 up to 2.3 at the $\mathrm{q}=3 / 2$ of scenario 3 ) and smaller $w_{c d}$ are obtained at the resonant surfaces of all scenarios (apart at the $\mathrm{q}=2$ surface of the low $\mathrm{q}$ Scenario 5 due to the above mentioned beam size effect); iii) still in the case of DUL, for all relevant surfaces the product $\eta_{N T M} w_{c d}$ is larger by a factor from $13 \%$ ( $\mathrm{q}=2$ of scenario 2 ) up to $39 \%$ ( $\mathrm{q}=3 / 2$ of scenario 3 ).

Since the power required for full stabilization, depending also on the width at which the mode is stabilized, decreases for smaller $w_{c d}$ and for larger values of the products $\eta_{N T M} w_{c d}$, we may argue that, by injecting the EC power from the DUL, a reduction of $\sim 20 \%$ (on average) of the power needed for stabilize the NTMs may be achieved. We also stress, however, that accurate evaluations should also take into account the marginal island width below which the modes self stabilize [18].

Concerning the capabilities of the two launchers for sawtooth control, it is clear that accurate predictions require transport modelling including a sawtooth period model that takes into account the stabilizing effect of alpha particles. However, a criterion to alter the shear at $\mathrm{q}=1$ for a significant change in the sawtooth period has been provided in the form $I_{E C C D} / \Delta \rho^{2} \geq I_{p}\left(\rho_{q=1}\right) / 2\left(\rho_{q=I}\right)^{2}, I_{E C C D}$ being the total driven current, $\Delta \rho$ the (normalized) full width at $1 / \mathrm{e}$ of the driven current profile, and $I_{p}\left(\rho_{q=1}\right)$ the plasma current inside the $\mathrm{q}=1$ surface [19]. Although this criterion does not take into account the stabilizing effect of alpha particles, so that it will not apply quantitatively to ITER, $\eta_{s}=I_{E C} / \Delta \rho^{2}$ is taken as figure of merit and used here to compare the EPL and DUL efficiencies for local shear modification. The values of $\eta_{s}$ for $\mathrm{P}_{\mathrm{EC}}=1 \mathrm{MW}$ injected from the DUL_USM and EPL_USM are shown in figure 4. According to these results, the DUL efficiency to alter the magnetic shear close to $q=1$ 
appears to be a factor $\sim 4$ superior to the EPL efficiency. However, to confirm that the DUL can increase the shear at $\mathrm{q}=1$ at a faster rate and to a larger value of EPL requires a full simulation.

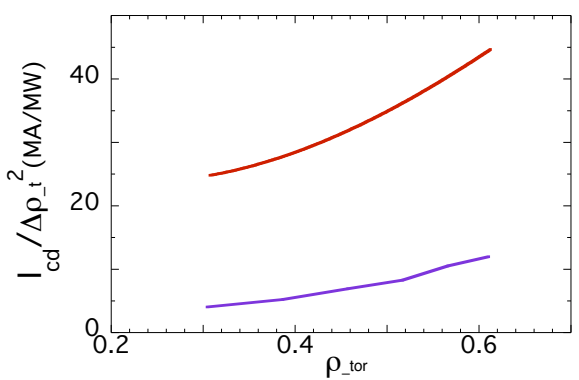

Figure 4. Values of the figure of merit for sawtooth control for $1 \mathrm{MW}$ injected in the plasma of scenario 2 from the DUL_USM (red curve) and from the EPL_USM (blue curve).

\section{Analysis and performances of the Equatorial Launcher}

The current design of the EL foresees 1 equatorial port, 24 beams injected from 3 steering mirrors ( 8 beams/mirror) located at $\mathrm{R}=9.2 \mathrm{~m}$ and $\mathrm{z}=0.02,0.62$ and $1.22 \mathrm{~m}$, which we shall call low, mid and top mirror, respectively. The launch is horizontal, with a toroidal steering capability between $20^{\circ}$ and $46^{\circ}$, and provides only co-current drive [20]. As already pointed out by several authors, see e.g. references $[21,22]$, only the mid-mirror can deposit the power close to the plasma centre, while central deposition may be improved by adding a fixed poloidal angle to the top and low front mirrors. In reference [17] it has been shown that a tilt angle $\alpha \sim \pm 10^{\circ}$ allows driving current very close to centre of the plasma of Scenario 2. To account for possible vertical shifts of the plasma centre, we performed the calculations shown here assuming a tilt angle of $\pm 5^{0}$ for the top and low front mirrors, respectively. To simulate the eight beams, a single wide astigmatic beam, characterized by the waists $w_{1}=4.04 \mathrm{~cm}$ and $w_{2}=1.02 \mathrm{~cm}$ at distances $d_{1}=-678.1 \mathrm{~cm}$ and $d_{2}=69 \mathrm{~cm}$ from the front mirrors (with suffix 1 indicating the horizontal axis, and suffix 2 the vertical axis in a plane orthogonal to the beam line) has been considered. The driven co-current, the peak current density, the normalized radius of the peak current density and the normalized width of the current density profiles as a function of the toroidal injection angle are shown in figure 5 for the scenario 2 and in figure 6 for the case of the hybrid scenario 3 .
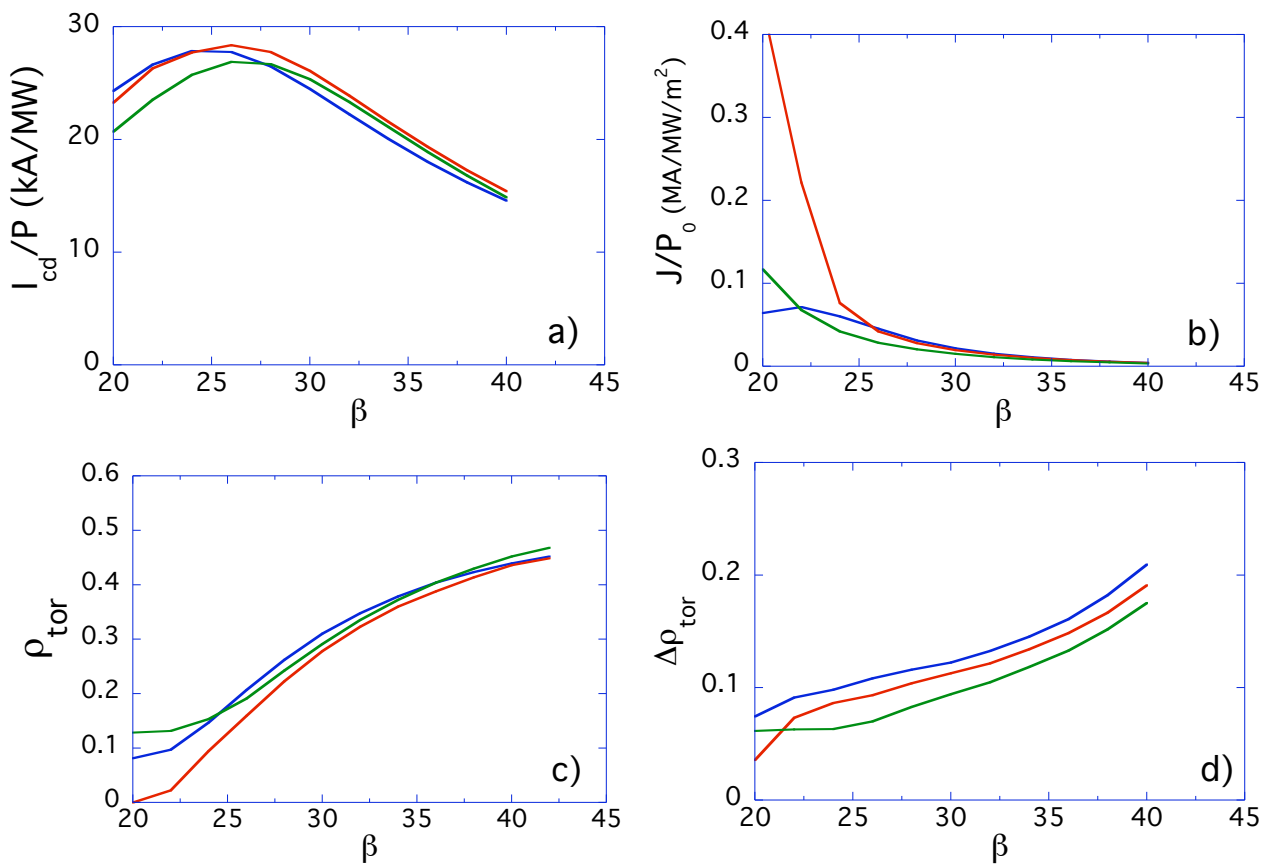

Figure 5. Driven co-current per unit power a), peak current density b) normalized radius of the peak current density c) and normalized width at $1 /$ e of the current density profile d) as a function of the toroidal steering angle 
for the three rows of the EL with a tilt angle of $5^{0}$ of the top and low front mirrors. The red curves refer to the mid-mirror, the blue ones to the low-mirror and green ones to the top-mirror. The equilibrium and plasma parameters are those of scenario 2 at EOB. The curves are plotted up to $\beta$ values that allow full power absorption.
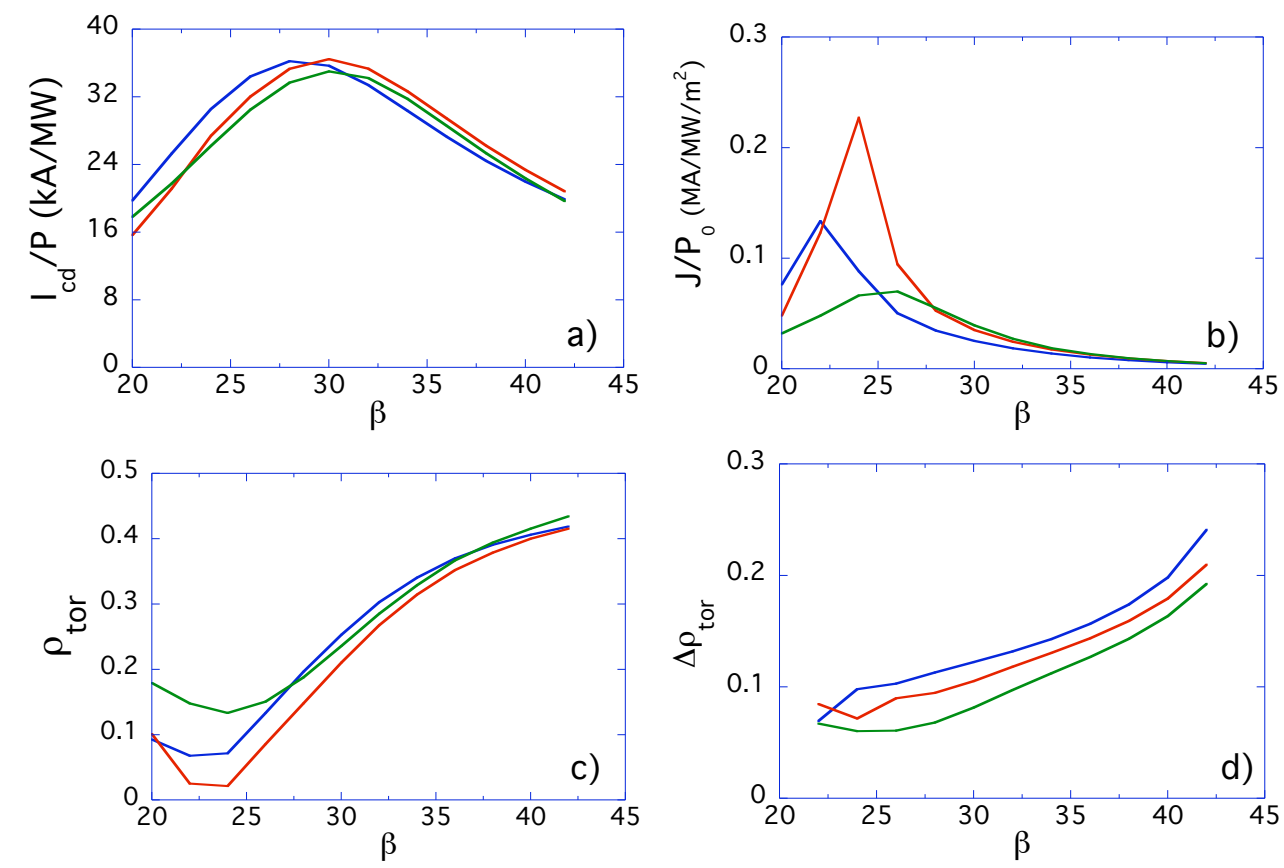

Figure 6. Same as in figure 5, for the plasma of scenario 3.

It may be observed that a large amount of co-current close to the plasma centre is driven for $\beta=20^{\circ}$, in the case of scenario 2 , and for $\beta \sim 23^{0}$ in the case of scenario 3, while quite broad current density profiles are obtained. Both the beam size and $\mathrm{n}_{/ /}$are seen to contribute broadening the current density profiles up to $\beta=30^{\circ}$, in scenario 2 , up to $\beta=28^{0}$ in scenario 3. For larger $\beta$ values, the Doppler shift prevails. Therefore, a beam with a smaller size would not improve the poor localization of the EC current driven at radii $\rho_{\text {tor }}>0.3$ in scenario $2, \rho_{\text {tor }}>0.2$ in scenario 3. Also to be noticed is that for high $\beta$ values $\left(\beta>40^{0}\right)$, high $\mathrm{n}_{/ /}$effects lead to incomplete absorption of the O-mode EC power. As a consequence, the access range for each mirror is limited up to $\rho_{\text {tor }}<0.5$, depending on the scenario. Moreover, for the low $\beta$ values for which the interaction with the O-mode first harmonic occurs on the high field side (this happens for scenario 3 at $\beta$ values below $22^{\circ}$ ), a fraction of the power may be absorbed at downshifted second harmonic, leading to ECCD profiles with a counter-current in addition to the driven co-current. An example of such profile is shown in figure 7 , in the case of the high temperature scenario 3 for injection at $\beta=20^{\circ}$. In the case shown in the figure, $40 \%$ of the power injected from the mid row is absorbed as second harmonic close to the plasma centre.

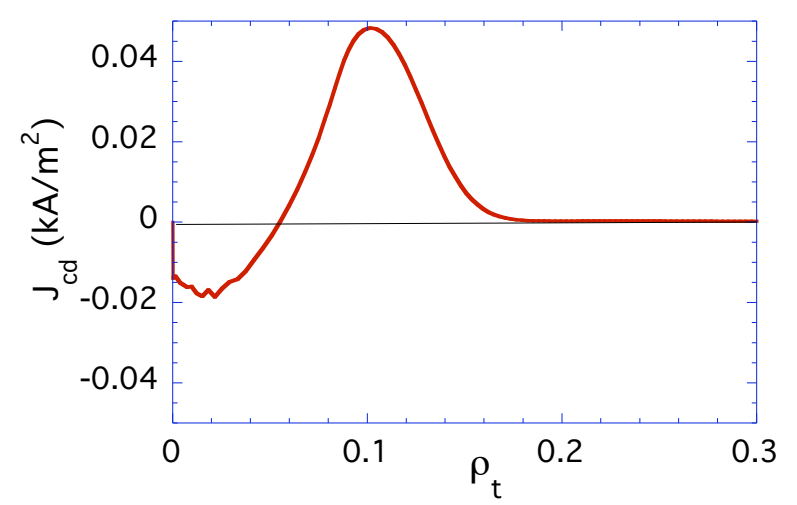

Figure7. Current density profile for $1 \mathrm{MW}$ EC power injected from the mid row into the plasma of scenario 3 , for $\beta=20^{0}$ 
In order to fulfil its physics objectives as core heating (without peaking the plasma current), sawtooth control, to assist a reversed shear formation or to avoid current holes, the EL design should allow also for counter current drive. Therefore, in addition to the inductive scenario 2 and the hybrid scenario 3, here we shall also consider the advanced scenario 4. The latter is a reverse shear scenario with an internal transport barrier. Profiles of co- and counter-ECCD computed for the three scenarios by taking into account the full available power $\mathrm{P}_{\mathrm{EC}}=20 \mathrm{MW}$ are shown in figure 8 . The toroidal angles $\beta$ have been chosen to get the ECCD profiles closest to the plasma centre in each scenario. The total driven current values are +0.45 MA and -0.47MA for scenario 2, +0.51 MA and -0.54 MA for scenario 3 and, +0.66 MA and -0.67 MA for scenario 4, where + refers to co-ECCD and - to counterECCD.
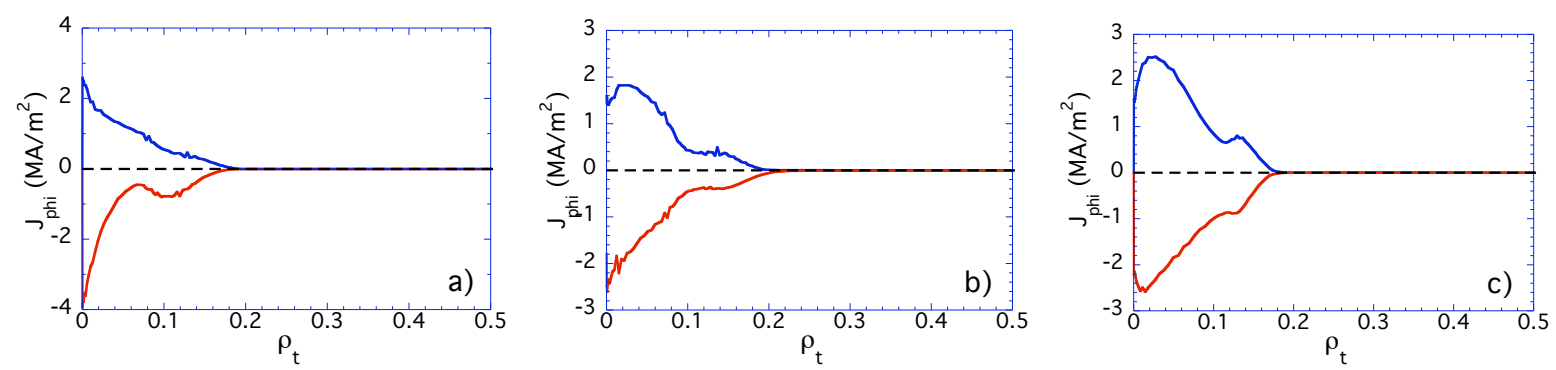

Figure 8. Co- and counter-ECCD profiles for $20 \mathrm{MW}$ injected from the EL with a tilt angle of $5^{0}$ of the top and low front mirrors, and a toroidal angle $\beta= \pm 20^{\circ}$ for Scenario 2 a), $\beta= \pm 23^{\circ}$ for Scenario $3 \mathrm{~b}$ ), $\beta= \pm 25^{0}$ for Scenario $4 \mathrm{c})$.

The potential effects of the driven current on the q profiles have been estimated by the equilibrium code CHEASE [23], and are shown in figure 9. Although the resistive evolution of the plasma current profile is not taken into account in the present analysis, results show that, with an improved design, the EL could provide a large range of variation of central q for all scenarios, particularly if counter-ECCD is included in the EL capabilities. It is also important to note that, whenever central heating is required, the driven co-current might peak the plasma current density profile and lead to early appearance of the $\mathrm{q}=1$ surface for the hybrid scenario or to a monotonic profile for Scenario 4 . The only possibility of avoiding unwanted low central $\mathrm{q}$ values is to compensate with a counter current to allow either central heating (co- + counter-CD) or central counter-CD. Even a single row of counter ECCD beams in the EL would significantly increase the potentialities of the system, providing a larger flexibility in controlling $\mathrm{q}_{0}$ in the advanced Scenario 4.


Figure 9. Potential effects of co-ECCD (blue region) and counter-ECCD (pink region) on the q profiles of Scenario 2 a), Scenario 3 b) and Scenario 4 c) estimated by CHEASE code. Dashed red lines refer to counterECCD obtained with 6.7 MW in case of Scenario 3, and with only $3 \mathrm{MW}$ in case of Scenario 4. Dashed blue lines indicate the potential effects of co-ECCD provided by $10 \mathrm{MW}$ for both cases of Scenario 3 and Scenario 4 .

\section{Analysis at reduced magnetic field}

The analysis presented so far has been carried out at the nominal value of the ITER magnetic field. However, at initial operation, ITER will likely work at magnetic fields $\mathrm{B}_{\mathrm{T}}<5.3 \mathrm{~T}$. The question therefore arises on determining the accessibility of the present system at reduced magnetic fields. The 
problem for the UL (based on three upper ports) was analyzed in [24, 25]. Although the magnetic topology of the equilibrium of scenario 2 was not identical and a different scaling of kinetic parameters was used, similar conclusions were drawn for the UL in the two papers. The analysis we present here has been performed for scenario 2 (EOB2), scaling the plasma current and kinetic profiles in order to keep fixed the edge safety factor $\mathrm{q}_{95}$, the plasma $\beta$ and the Greenwald number $\mathrm{n} / \mathrm{n}_{\mathrm{G}}$.

It is worth recalling that neither Upper nor Equatorial launchers can work in the magnetic field range $3.4 \mathrm{~T}-4.2 \mathrm{~T}$ since the EC resonances for $\mathrm{f}=170 \mathrm{GHz}$ are located outside the plasma or quite close to the boundary. For the UL, where the toroidal angle $\beta$ is fixed, when the magnetic field is reduced below $5.3 \mathrm{~T}$ for case of O-mode, first harmonic (OM1), and below 2.65 T, for the case of X-mode second harmonic (XM2), it is generally observed that the access to innermost flux surfaces is strongly reduced whenever the EC resonance is too far in the high field side. In the case of XM2, for magnetic fields beyond $2.65 \mathrm{~T}$ low and broad current density profiles are obtained when the EC resonance falls close to the radial location of the launcher. This is due to both trapping effects and to the 'quasi' vertical trajectories (with high $\alpha$ values) required to drive current at the relevant flux surfaces.

In the case of the EL, where the toroidal angle $\beta$ is used to steer the ECCD, when $B_{T}$ decreases with respect to $5.3 \mathrm{~T}$, the OM1 power absorption and ECCD occurs in the high field side with respect to the plasma centre. On these long trajectories, a fraction of the EC power may be absorbed at downshifted second harmonic, leading to ECCD profiles with a counter-current in addition to the driven co-current. For magnetic fields around $2.65 \mathrm{~T}$ (XM2 case), finally, third harmonic absorption (XM3) may considerably reduce the EC power available close to the second harmonic resonance.

Let then investigate if advantages can be found, in terms of accessibility and performances in plasmas at reduced magnetic fields using the DUL instead of the EPL.

The OM1 case is represented in figures 10 and 11, where the driven current density as a function of its radial location is shown for magnetic fields reduced by $10 \%, 15 \%$ and $20 \%$ with respect to the nominal field. As seen, ECCD down to $\rho_{\text {tor }}<0.5$ is no more allowed for both the DUL_USM and the EPL_USM, already for a $10 \%$ reduction of the nominal magnetic field. The possibility of using the USM for sawtooth control is therefore lost in both cases. In order to determine the allowed $\mathrm{B}_{\mathrm{T}}$ range, we use the criterion that co-current has to be driven in all the radial range of NTMs, i.e. from $\rho_{\text {tor }}=0.55$ up to $\rho_{\text {tor }} \leq 0.85$, at least by launching from the LSM. As easily argued from figure 10, the minimum field for which the above criterion is satisfied is $\mathrm{B}_{\mathrm{T}} \sim 4.5 \mathrm{~T}$ for the DUL, and $\mathrm{B}_{\mathrm{T}} \sim 4.7 \mathrm{~T}$ for the EPL. Therefore the $B_{T}$ range where OM1 current drive is efficient for stabilization of all NTMs is larger for DUL. Furthermore, recalling that the bootstrap current density is scaled as the magnetic field, we also find that at resonant surfaces of scenario EOB2 the values of the NTM figure of merit, for a power of 13.3 MW, exceeds the threshold value 1.2 in the whole range of magnetic field available for each launcher.

The same analysis has been performed for XM2 around half the nominal magnetic field, $\mathrm{B}=2.65 \mathrm{~T}$. The range of magnetic fields where the two launchers efficiently drive current is from $2.3 \mathrm{~T}$ to $2.8 \mathrm{~T}$ for the EPL, and from $2.3 \mathrm{~T}$ up to $3 \mathrm{~T}$ for the. Therefore the magnetic field gap between OM1 and XM2 is reduced by $20 \%$ if the EC power is launched from the DUL.
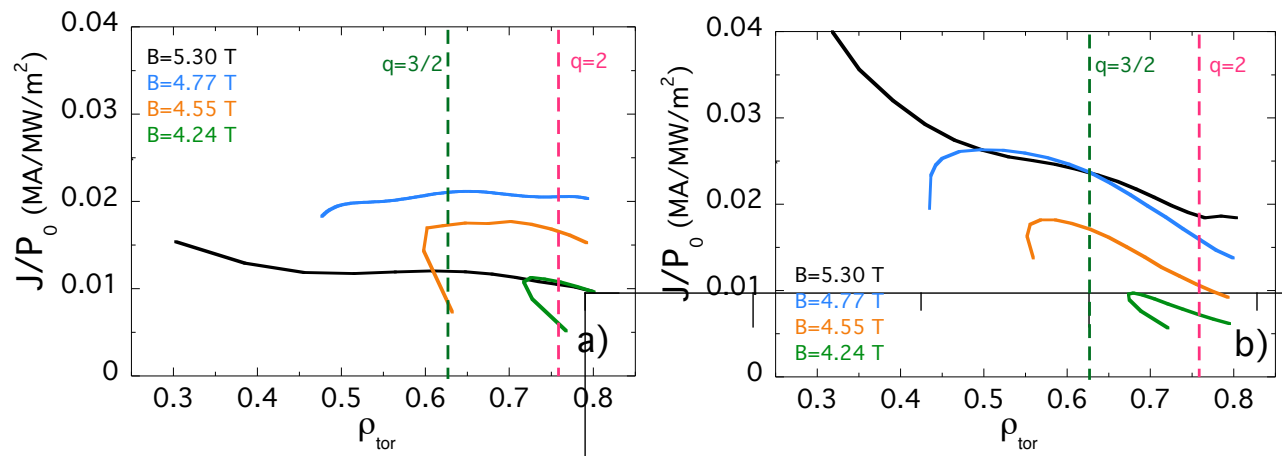

Figure 10. Driven current density per unit EC power, for magnetic fields reduced by $10 \%, 15 \%$ and $20 \%$, for injection from the USM of the EPL a), for injection from the USM of the DUL b). The nominal field case is 
included (black curves) as a reference. The vertical dashed lines indicate the radial location of $\mathrm{q}=3 / 2$ surface (green) and q=2 surface (pink) for scenario EOB2.
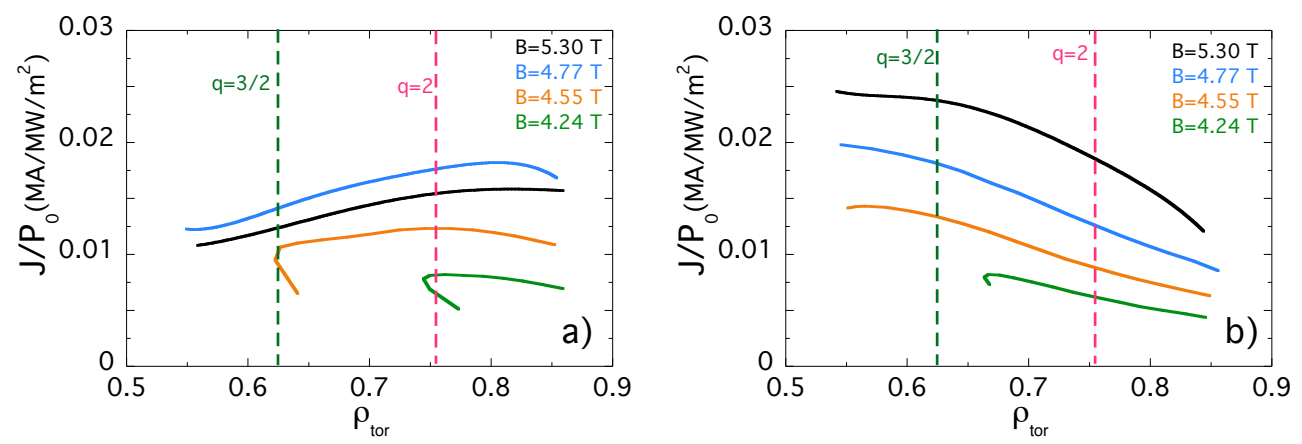

Figure 11. Same as figure 10, for injection from the EPL_LSM a), for injection from the DUL_LSM b).

The capabilities of the EL at reduced $\mathrm{B}_{\mathrm{T}}$ have been investigated by considering the mid row only. The results obtained for the OM1 case are shown in figure 12 , for values of $\mathrm{B}_{\mathrm{T}}$ reduced by $10 \%, 15 \%$ and $20 \%$. It is worth pointing out that, as $\mathrm{B}_{\mathrm{T}}<5.3 \mathrm{~T}$, the effective $\beta$ range decreases due to incomplete power absorption. As already mentioned, at the low $\beta$ values for which co-current is driven in the high field side with respect to the plasma centre, a counter-current affects the driven current profiles. This occurs at $\beta<25^{0}$ for $\mathrm{B}_{\mathrm{T}}=4.77 \mathrm{~T}$, at $\beta<27^{0}$ for $\mathrm{B}_{\mathrm{T}}=4.55 \mathrm{~T}$ and at $\beta<32^{0}$ for $\mathrm{B}_{\mathrm{T}}=4.24 \mathrm{~T}$. Moreover we note that the access range is limited up to $\rho_{\text {tor }} \leq 0.2$ for $\mathrm{B}_{\mathrm{T}}=4.77 \mathrm{~T}$, and up to $\rho_{\text {tor }} \leq 0.3$ for $\mathrm{B}_{\mathrm{T}}=4.55 \mathrm{~T}$.
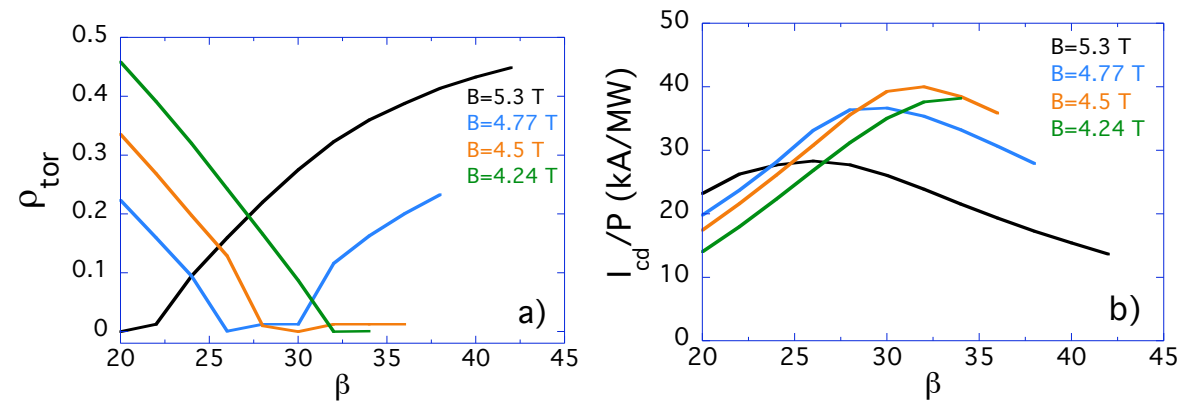

Figure 12. Radial location a) and total driven current b) as a function of $\beta$, by injecting $1 \mathrm{MW}$ of EC power as OM1 from the mid-row of the EL at nominal field (black curves) and three values of $\mathrm{B}_{\mathrm{T}}$ reduced by $10 \%, 15 \%$ and $20 \%$. The curves are shown up to $\beta$ values that allow full power absorption.

The performances of the EL for injection of second harmonic X-mode (XM2) are shown in figure 13.
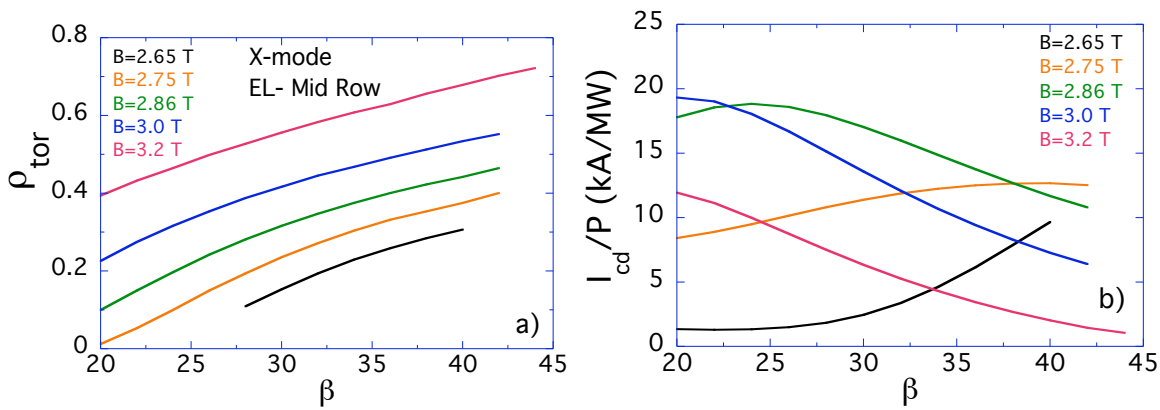

Figure 13. Same as figure 13, in the range of B from $2.65 \mathrm{~T}$ up to $3.2 \mathrm{~T}$.

As already mentioned, we observe that, for $\mathrm{B}_{\mathrm{T}} \leq 2.75 \mathrm{~T}$, third harmonic effects become dominant. A large fraction of the injected power is absorbed as XM3 in the low field side region, even preventing the interaction in the XM2 region, and the values of the driven current become quite low. This result, however, is strongly dependent on the assumption one makes for the temperature at scaled magnetic 
field. We also observe that a significant amount of current may be driven in the central region only for $\mathrm{B}_{\mathrm{T}}$ in the range $2.75 \mathrm{~T}--3 \mathrm{~T}$. For $\mathrm{B}_{\mathrm{T}}$ increasing from $3 \mathrm{~T}$ to $3.2 \mathrm{~T}$, the radial range where a significant amount of current may be driven is shifted towards large radii, therefore the main goals of the EL, i.e. heating and $\mathrm{CD}$ close to the centre, can no more be fulfilled. We finally observe that for $\mathrm{B}_{\mathrm{T}}>3.2 \mathrm{~T}$, negligible current is driven at outermost surfaces since ECCD is dominated by trapped particle effects.

\section{Summary and conclusions}

The analysis performed in this work compared the performance of the present EC Upper Launcher design, the EPL launcher, with that of the same launcher moved vertically down in the ITER machine by approximately one blanket shield module, labelled the DUL launcher. The comparison showed that the DUL, by providing higher and narrower current density profiles, would have increased performances for both NTM stabilization and sawtooth control. The better performances for DUL occur mainly at innermost surfaces, leading to an increment of the figure of merit for sawtooth control up to a factor 4. An exception to the favourable behaviour is found for the EC current driven at outermost surfaces, and is mainly due to the fact that the beam used for the LSM in the present simulation is not 'optimized' for the DUL. Another advantage of lowering the upper port plug is that, at low $\mathrm{B}_{\mathrm{T}}$ operation, the range of magnetic field where EC current may be driven in the whole radial range of NTMs would increase both in the OM1 case and in the XM2 case. Therefore the magnetic field range where the NTMs region is not accessible would be reduced by $20 \%$ with respect to the present location. The main disadvantage of the DUL is that it requires a larger steering range to drive current in the same plasma region compared to the EPL. This would result in a larger rotation of the steering mirror and, as a consequence, in higher levels of induced stress in the critical component of the steering mechanism.

The main evidences provided by the analysis performed for the Equatorial Launcher are that, with a tilt angle of 5 degrees added to the top and low front mirrors, a better access to the plasma core can be provided. However the access range is limited to $\rho_{\text {tor }}<0.5$, due to incomplete power absorption for the O-mode at large $\mathrm{n}_{/ /}$values. Quite broad current density profiles $\left(\Delta \rho_{\mathrm{t}}>0.1\right)$ are observed in the radial region where the $\mathrm{q}=1$ surface is likely to occur, i.e., $\rho_{\text {tor }} \sim 0.3-0.4$, the broadening being dominated by the Doppler shift. This makes the EL less efficient than the UL for sawtooth control. Moreover it has been shown that, adding to present design the possibility to drive counter-current in addition to the existing co-current capability, would largely increase the flexibility of the system, by decoupling heating and current drive, whenever only heating is necessary, or providing the possibility of controlling the central $\mathrm{q}$ in the advanced Scenario 4.

Finally, it has been shown that the EL has a very limited access range (up to $\rho_{\text {tor }} \leq 0.2$ ) when the magnetic field is reduced by only $10 \%$. Recalling that, for the same magnetic field, the UL cannot drive current inside $\rho_{\text {tor }}=0.5$, sawtooth control is not anymore possible at this field. For a magnetic field reduced by $15 \%$ or by $20 \%$, the access range of the EL increases up to $\rho_{\text {tor }} \sim 0.3$ or up to $\rho_{\text {tor }} \sim$ 0.45 , respectively, however the current density profiles show also a counter current due to power absorption at downshifted second harmonic. Moreover, in the range of second harmonic X-mode, a significant amount of current may be driven in the central region only for $\mathrm{B}_{\mathrm{T}}$ in the range $2.75 \mathrm{~T}-\mathrm{-} 3 \mathrm{~T}$. Therefore the capabilities of the EL are strongly reduced by reducing the magnetic field.

\section{ACKNOWLEDGMENTS}

This work, supported by the European Community, was carried out within the framework of the European Fusion Development Agreement (TASK TW6-TPHE). The views and opinions expressed herein do not necessarily reflect those of the European Commission. Some of the authors are supported in part by the Swiss National Science Foundation.

\section{References}

[1] Weisen H et al., 2006 Plasma Physics Control Fusion 48 A457-A466

[2] Farina D 2007 to appear on Fusion Science and Technology 52

[3] Poli E, Peeters A G and Pereverzev G V 2001 Comp. Phys. Comm. 13690

[4] Zohm H et al., 2005 Journal of Physics: Conference Series 25234 
[5] La Haye R J et al., 2006 Nuclear Fusion 46, 451

[6] Saibene $\mathrm{G}$ et al., 2006 Proc. 21 st IAEA Fusion Energy Conference, IT/P2-14

[7] Henderson $\mathrm{M}$ et al, 2006 Proc. 21st IAEA Fusion Energy Conference, IT/P2-15

[8] Marasheck M et al., 2004 Proc. 19th IAEA Fusion Energy Conference, EX-7/2

[9] Porcelli F, Boucher D and Rosenbluth M N, 1996 Plasma Phys. Control. Fusion 382163

[10] Sauter O et al., 2002 Physical Review Letters 88, 105001

[11] Angioni C et al., 2003 Nuclear Fusion 43, 455

[12] Eriksson L G, 2004 Physical Review Letters 92, 235004-1

[13] J.P. Graves et al., 2005 Plasma Physics Control Fusion 47, B121-B123

[14] Sauter O, Zohm H, 2005, Proceedings of the 26th EPS Conf. on Plasma Physics, 29C P-2.059

[15] Harvey R V, Perkins F V, 2001 Nuclear Fusion 41,1847

[16] Ramponi G et al., 2003, Proceedings of the 2nd IAEA Technical Meeting on ECRH for ITER

[17] Ramponi G et al., 2007 to appear on Fusion Science and Technology 52

[18] Sauter O et al., 2006, Proceedings of 21st IAEA Fusion Energy Conference, TH/P3-10

[19] Merkulov A et al., 2004, Proceedings of the Joint Varenna-Lausanne International Workshop on Theory of Fusion Plasmas, 279

[20] Takahashi K et al, 2005 Journal of Physics: Conference Series 25,75

[21] Volpe F, 2005 Journal of Physics: Conference Series 25, 283

[22] Prater R, 2005 Journal of Physics: Conference Series 25, 257

[23] Ramponi G, Farina D and Nowak S, 2005 Journal Physics: Conf. Series 25, 243 